\title{
Dural Arteriovenous Fistula Manifested as Rapid Progressive Dementia Successfully Treated by Endovascular Embolization Only
}

\author{
Heewon Hwang, MD', Yun Kyung La, MD', Min Seok Baek, MD', Kyoungwon Baik, MD', \\ Sang Hyun Suh, MD, PhD², Won-Joo Kim, MD, PhD'
}

A 43-year-old male presented with daytime sleepiness at work and indifferent behavior like never before. Two weeks prior to hospital admission, he had episodic memory loss with well preserved remote memory. Brain MRI showed a dural arteriovenous fistula (DAVF) in the right lateral transverse sinus with a bilateral thalamic venous infarction. Cerebral angiography confirmed a right transverse sigmoid dural arteriovenous fistula with a feeding artery of the right occipital artery and left posterior meningeal artery. The DAVF was completely eliminated through multiple endovascular interventions. Recently, endovascular treatment has become one of the main therapeutic options to obliterate a fistulous site, which has led to a rapid diagnostic approach and management of DAVFs with high curative rates. We report a rare case of posterior fossa located at a dural arteriovenous fistula that caused rapid progressive dementia and was successfully eliminated through only endovascular treatment.

Key Words : Dural arteriovenous fistula; Dementia; Therapeutic embolization

A dural arteriovenous fistula (DAVF) is an uncommon type of intracranial vascular malformation. Though the true incidence of DAVF is unknown, the reported incidence is approximately $10-15 \%$ of all intracranial vascular malformations [1]. Clinical manifestations of intracranial DAVF are various includ-

\footnotetext{
Departments of ${ }^{1}$ Neurology and ${ }^{2}$ Radiology, Gangnam Severance Hospital, Yonsei University College of Medicine, Seoul, Korea Received January 31, 2016;

accepted after revision February 14, 2017.

Correspondence to: Won-Joo Kim, MD, PhD, Department of Neurology, Gangnam Severance Hospital, Yonsei University College of Medicine, Eonjuro 211, Gangnam-gu, Seoul 06273, Korea

Tel. 82.2.2019.3320 Fax. 82.2.3462.5904

E-mail: kzoo@yuhs.ac

This is an Open Access article distributed under the terms of the Creative Commons Attribution Non-Commercial License (http://creativecommons.org/licenses/by-nc/3.0) which permits unrestricted non-commercial use, distribution, and reproduction in any medium, provided the original work is properly cited.
}

ing pulsatile tinnitus, orbital congestion, headache, intracranial hemorrhage or infarction [2]. However, there have been little reports about DAVFs manifested as rapidly progressive dementia [3,4]. Most offending DAVFs causing dementia are located at the tentorial edge, thereby a report about DAVFs located in the posterior fossa has meaning.

We report a case of a DAVF located in the posterior fossa that manifested as a rapidly progressive dementia and was completely eliminated by endovascular embolization alone.

\section{CASE REPORT}

A 43-year-old male was brought to the emergency department due to inattention and transient episodic memory loss. He was relatively healthy, except for taking medications for a headache that developed a year ago. For 2 weeks prior to admission, he showed 


\section{Dural Arteriovenous Fistula with Rapid Progressive Dementia}

excessive daytime sleepiness at work and an indifferent mood noticed by his colleague during a conference. One day before admission, the patient got lost going back home from work. It took more than 2 hours to get home, which normally took 30 minutes. On the day of admission, the patient showed transient episodic memory loss of that day. He had intact remote memory. Neurologic examination demonstrated no other lateralizing of focal deficit. His initial Korean mini mental status exam (K-MMSE) score was 27 out of 30 , showing a mild impairment in attention. On the following day, K-MMSE score had fallen to 19 out of 30 , showing a worsening of attention and mild impairment in memory recall. The electroencephalogram was normal. A cerebral spinal fluid study showed high opening pressure of $330 \mathrm{mmH} 2 \mathrm{O}$, but was acellular showing a nonspecific finding. Brain MRI showed a DAVF in right lateral sinus with a bilateral thalamic venous infarction (Fig. 1A, F). The following cerebral angiography confirmed a right transverse sigmoid DAVF with a feeding artery of the right occipital artery and left posterior meningeal artery (Fig. 1B, C). Endovascular embolization was performed three times due to a highly stenotic, compartmentalized sinus and small, tortuous vessels (Fig. 1D, E). After the third intervention, there was a decrease in extent of venous congestion in the bilateral thalami. Follow up Brain MRI taken one month later after embolization showed a remaining DAVF, though with a decreased signal change in the bilateral thalami. At the time of discharge, the follow-up K-MMSE score showed improvement with a score of 27 out of 30 with improvement in attention and memory recall.

The fourth embolization was performed 5 months after initial endovascular therapy. Since there were some thrombus at the sagittal sinus and straight sinus, we decided to maintain warfarin anticoagulation. At the time of discharge, his mental status and mental function
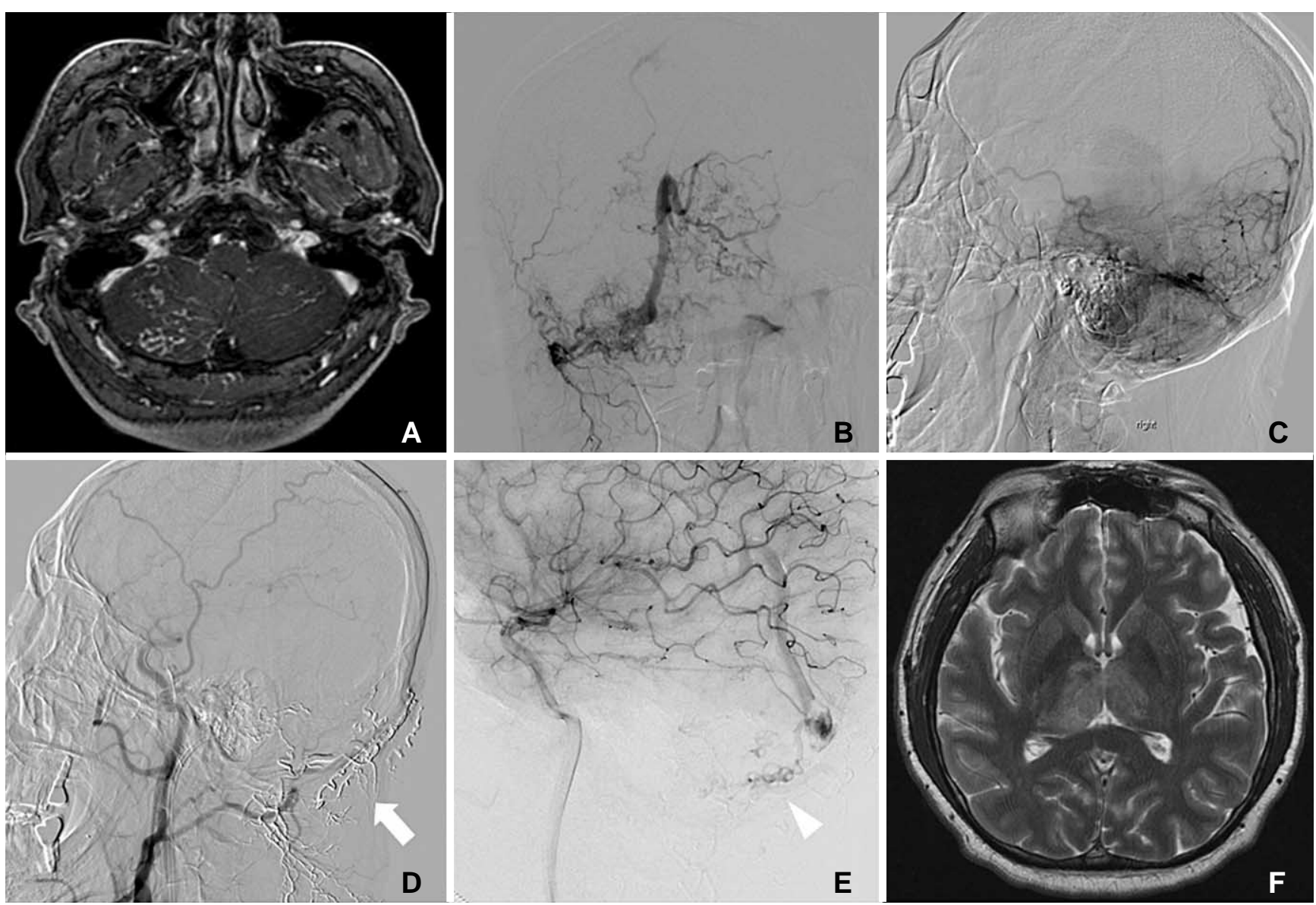

Fig. 1. Brain magnetic resonance imaging (MRI) shows a DAVF in the right lateral sinus with numerous feeding arteries in the posterior fossa $(\mathbf{A})$. Cerebral angiography coronal view $(\mathbf{B})$ and sagittal view $(\mathbf{C})$ shows a right transverse sigmoid DAVF with a feeding artery of the right occipital artery and left posterior meningeal artery. Embolization was performed through two transmastoid feeders from the left occipital artery (white arrow)(D). Additional DAVF embolization was performed in the remnant fistulas of the right middle meningeal artery and left ICA-hypophyseal trunk via the right middle meningeal artery (white arrowhead)(E). Initial brain MRI shows a bilateral thalamic T2 signal change $(\mathbf{F})$. 


\section{Heewon Hwang, et al.}

was well preserved and the patient had begun his normal daily life. Follow up cerebral angiography performed 1 year later showed no evidence of a residual AVF.

\section{DISCUSSION}

DAVFs manifest various symptoms in relation to the location of the lesion and pattern of venous drainage. If blood flow increases in dural venous sinuses, especially in the transverse and sigmoid sinus, the most common symptom is known as pulsatile tinnitus. Furthermore, a DAVF in the cavernous sinus has symptoms of ophthalmoplegia, proptosis, retro-orbital pain and decreased visual acuity. There can be other neurologic deficits according to the various locations of the DAVF, such as seizure, parkinsonism and cerebellar symptoms [5].

As in our case, atypical symptoms, such as rapidly progressive dementia, can occur. Venous congestion and ischemia of the thalamus occurred through venous hypertension followed by retrograde cerebral blood flow that caused thalamic dementia. As far as we know, a DAVF case with cognitive impairment and transient memory loss is uncommon [3,6-8]. In our case, the patient had bilateral thalamic congestion due to a DAVF located in the posterior fossa and showed episodic memory loss with inattention and disorientation. This case is atypical since usually a tentorial DAVF with cortical venous drainage tends to offend the bilateral thalami and cause dementia. These bilateral thalamic involvements also cause deficits in learning, attention and executive function. The clinical feature of our patients could be thought as having memory impairment and inattention owing to the lesion involving the mamillothalamic tract that leads to neurocircuit dysfunction of limbic system.

Patient with a DAVF of benign symptoms and a lowgrade lesion can be managed with conservative care but some patients with a severe disease and complications need prompt endovascular repair [9]. On the other hand, for patients with rapidly progressive dementia, prompt management of a DAVF is important before further deterioration of cognitive function. Therefore, early intervention through surgery or endovascular embolization is important. Since a surgical approach could be more invasive and provoke severe complications such as hydrocephalus or hemorrhage, as in our case, complete obliteration of the DAVF can done by endovascular treatment alone. The vast majority of DAVFs show a highly stenotic, compartmentalized, and isolated sinus and a relatively small, tortuous cortical vein. Thereby, embolization with a transarterial approach should be the option chosen. Transarterial embolization (TAE) makes selective distal catheterization of arterial feeders possible through embolic agents such as particles, coils, ethanol, n-BCA glue and Onyx. However, the successful elimination a DAVF by TAE is known to be rare because DAVFs often involve multiple, small cerebral arteries that are difficult to approach [10]. After a single endovascular treatment, additional feeders may appear that results in a partially treated status that could later provoke complications such as hemorrhage. Therefore, absolute elimination of a DAVF is crucial if it's decided to manage a DAVF through TAE. In our case, even though multiple interventions were necessary due to a highly stenotic and tortuous vessel, complete elimination was achieved through endovascular approach alone. Oh et al. [11], had recently reviewed 13 cases of endovascular treatment of a DAVF in Korea. Eight cases were successfully treated by TAE alone with onyx. Other cases with an inaccessible route of the endovascular approach were treated through a combined surgical approach. As in this case series, it is already known that endovascular treatment alone can achieve complete remission of a DAVF. In addition to this, our case showed that prompt diagnosis and treatment of a DAVF manifesting a rapidly progressive dementia can achieve a good therapeutic result without surgical intervention.

As a conclusion, thalamic dementia caused by a DAVF located in posterior fossa is rare, but delayed recognition of its presence could cause irreversible ischemic damage of the thalamus. So evaluation of a DAVF located in the posterior fossa in patients showing thalamic dementia is important. In addition to emphasizing the importance of a quick diagnosis, our case showed that endovascular therapy alone can completely obliterate a DAVF located in the posterior fossa.

\section{References}

1. Chaichana KL, Coon AL, Tamargo RJ, Huang J. Dural arteriovenous fistulas: epidemiology and clinical presentation. Neurosurg Clin N Am 2012;23:7-13

2. Miller TR, Gandhi D. Intracranial Dural Arteriovenous Fistulae: Clinical Presentation and Management Strategies. Stroke 2015; 46:2017-2025

3. Holekamp TF, Mollman ME, Murphy RK, Kolar GR, Kramer NM, Derdeyn CP, et al. Dural arteriovenous fistula-induced thalamic dementia: report of 4 cases. J Neurosurg 2016;124:17521765

4. Morparia N, Miller G. Rabinstein A. Lanzino G. Kumar N. 


\section{Dural Arteriovenous Fistula with Rapid Progressive Dementia}

Cognitive decline and hypersomnolence: thalamic manifestations of a tentorial dural arteriovenous fistula (dAVF). Neurocrit Care 2012;17:429-433

5. Gandhi D, Chen J, Pearl M, Huang J, Gemmete JJ, Kathuria S. Intracranial dural arteriovenous fistulas: classification, imaging findings, and treatment. AJNR Am J Neuroradiol 2012;33:10071013

6. Hurst RW, Bagley LJ, Galetta S, Glosser G, Lieberman AP, Trojanowski J, et al. Dementia resulting from dural arteriovenous fistulas: the pathologic findings of venous hypertensive encephalopathy. AJNR Am J Neuroradiol 1998;19:1267-1273

7. Matsumura A, Oda M, Hozuki T, Imai T, Shimohama S. Dural arteriovenous fistula in a case of dementia with bithalamic MR lesions. Neurology 2008;71:1553
8. Lasjaunias P, Chiu M, Brugge KT, Tolia A, Hurth M, Berenstein M. Neurological manifestations of intracranial dural arteriovenous malformations. J Neurosurg 1986;64:724-730

9. Van Dijk JM, Willinsky RA. Venous congestive encephalopathy related to cranial dural arteriovenous fistulas. Neuroimaging Clin N Am 2003;13:55-72

10. Jabbour P, Tjoumakaris S, Chalouhi N, Randazzo C, Gonzalez LF, Dumont A, et al. Endovascular treatment of cerebral dural and pial arteriovenous fistulas. Neuroimag Clin N Am 2013;23:625636

11. Oh JS, Yoon SM, Oh HJ, Shim JJ, Bae HG, Lee KS. Endovascular Treatment of Dural Arteriovenous Fistulas: Single Center Experience. J Korean Neurosurg Soc 2016;59:17-25 